\title{
Coexistence of vortex arrays and surface capillary waves in spinning prolate superfluid ${ }^{4} \mathrm{He}$ nanodroplets
}

\author{
Martí Pi $\odot,{ }^{1,2}$ José María Escartín $\odot,{ }^{3}$ Francesco Ancilotto $\odot,{ }^{4,5}$ and Manuel Barranco ${ }^{1,2}$ \\ ${ }^{1}$ Departament FQA, Facultat de Física, Universitat de Barcelona, Avenida Diagonal 645, 08028 Barcelona, Spain \\ ${ }^{2}$ Institute of Nanoscience and Nanotechnology, Universitat de Barcelona, 08028 Barcelona, Spain \\ ${ }^{3}$ Catalan Institute of Nanoscience and Nanotechnology, CSIC and BIST, Campus UAB, Bellaterra, 08193 Barcelona, Spain \\ ${ }^{4}$ Dipartimento di Fisica e Astronomia “Galileo Galilei” and CNISM, Università di Padova, via Marzolo 8, 35122 Padova, Italy \\ ${ }^{5}$ CNR-IOM Democritos, via Bonomea 265, 34136 Trieste, Italy
}

(Received 15 April 2021; revised 28 August 2021; accepted 31 August 2021; published 9 September 2021)

\begin{abstract}
Within density functional theory, we have studied the interplay between vortex arrays and capillary waves in spinning prolate ${ }^{4} \mathrm{He}$ droplets made of several thousand helium atoms. Surface capillary waves are ubiquitous in prolate superfluid ${ }^{4} \mathrm{He}$ droplets, and depending on the size and angular momentum of the droplet, they may coexist with vortex arrays. We have found that the equilibrium configuration of small prolate droplets is vortex free, evolving towards vortex hosting as the droplet size increases. This result is in agreement with a recent experiment [O'Connell et al., Phys. Rev. Lett. 124, 215301 (2020)] that disclosed that vortex arrays and capillary waves coexist in the equilibrium configuration of very large drops. In contrast to viscous droplets executing rigid-body rotation, the stability phase diagram of spinning ${ }^{4} \mathrm{He}$ droplets cannot be universally described in terms of dimensionless angular momentum and angular velocity variables: Instead, the rotational properties of superfluid helium droplets display a clear dependence on the droplet size and the number of vortices they host.
\end{abstract}

DOI: 10.1103/PhysRevB.104.094509

\section{INTRODUCTION}

Superfluid ${ }^{4} \mathrm{He}$ droplets produced in the expansion of a cold helium gas [1] or by hydrodynamic instability of a liquid helium jet passing through the nozzle of a molecular beam apparatus [2] have been considered the ultimate inert matrix for molecular spectroscopy [3,4], constituting also an ideal test ground to study superfluidity at the nanoscale [5-11]. A series of experiments aiming at determining the appearance of large spinning ${ }^{4} \mathrm{He}$ droplets made of $10^{8}-10^{11}$ atoms [12-18] has motivated renewed interest in aspects such as how vortices are distributed inside helium droplets [19,20], how impurities are captured by the vortex lines they host, and how impurities arrange inside vortex-hosting droplets [21-27].

Two experimental techniques have been used to address large spinning ${ }^{4} \mathrm{He}$ droplets. The pioneering work in Ref. [12] and other works carried out by the same group $[13,14,18]$ used coherent diffractive imaging of $\mathrm{x}$ rays from a free-electron laser (FEL) and gave access to a model-independent determination of the two-dimensional (2D) projection of the droplet density on a plane perpendicular to the x-ray incident direction via iterative phase retrieval algorithms; by doping He droplets with Xe atoms they were also able to detect the presence of vortex arrays. In Refs. $[15,16]$, He droplets were irradiated with intense extreme ultraviolet pulses (XUV), and the measurements of wide-angle diffraction patterns provided access to full three-dimensional information. However, it was not possible to directly determine the shapes of the droplets, so the analysis was carried out parametrizing the droplet density with a combination of two ellipsoidal caps connected by a hyperboloidal centerpiece, and all their defining parameters were determined by matching the experimental diffraction patterns with those obtained from simulations.

These remarkable experiments have shown that most helium droplets are spherical and only a few percent are deformed. In particular, the analysis carried out by Langbehn et al. [16] on a sample consisting of a large number of droplets (38 150) showed that $92.9 \%$ of them were spherical, 5.6\% were spheroidal (oblate [28]), and $1.5 \%$ were prolate. The latter population was made of ellipsoidal $(0.8 \%)$, pill-shaped $(0.6 \%)$, and dumbbell-like $(0.1 \%)$ droplets, where the appearance was quantitatively determined by evaluating the distance of the center of mass of the droplet to its surface along the direction of the principal axes of inertia. The results obtained with both experimental techniques were compared to calculations made for incompressible viscous droplets subject to only surface tension and centrifugal forces [29-32]; it was concluded that they were in agreement.

The precise meaning of this agreement needs some clarification, as otherwise one might be left with the impression that the rotational properties of superfluid ${ }^{4} \mathrm{He}$ and of classical rotating viscous fluids are very similar, and that is not the case $[33,34]$. The experiments have, indeed, shown that the shapes of spinning superfluid ${ }^{4} \mathrm{He}$ drops are the same as those found for viscous drops subject to centrifugal and surface tension forces and that some relationships between ratios of the mentioned distances are in agreement with the classical results [32] (see, e.g., Fig. 3 of Ref. [16] and our Fig. 1). However, to ascertain whether the sequence of shapes is the same, which is what determines the equilibrium phase 


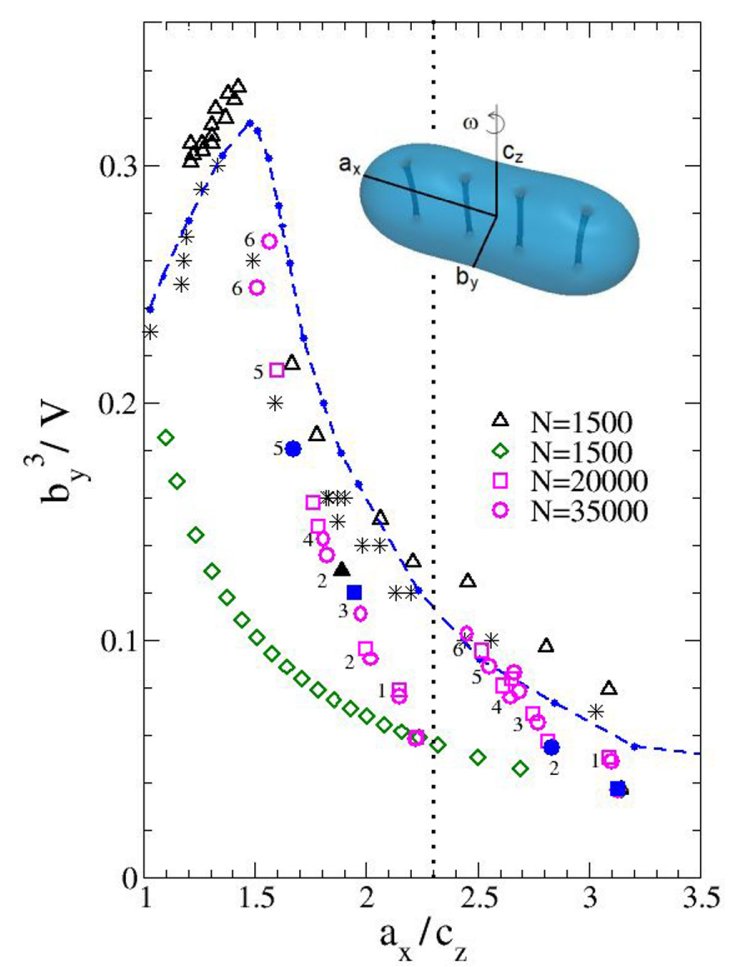

FIG. 1. Aspect ratio $b_{y}^{3} / V$ vs $a_{x} / c_{z}$ curve. Black triangles: Threevortex ${ }^{4} \mathrm{He}_{1500}$ configurations [20]. Green diamonds: Vortex-free (capillary wave) ${ }^{4} \mathrm{He}_{1500}$ configurations [20]. Magenta squares (circles): $N=20000$ (35000) configurations. The numbers close to the symbols indicate the $n_{v}$ value. The vertical dotted line separates the $N=20000$ and 35000 droplet configurations with $\Lambda=0.8$ (left) from those with $\Lambda=1.25$ (right). Solid symbols represent the global equilibrium configurations displayed in the tables. Black stars are the experimental results of Ref. [16], and blue dots connected by a dashed line are the classical rotating drop results [31,32]. The inset displays the lengths $a_{x}, b_{y}$, and $c_{z}$ used to characterize the droplet shape.

diagram, it is necessary to experimentally determine the angular momentum for a large enough sample of droplets; this has not been possible yet. Only very recently have the angular momentum and shape of a few He drops been simultaneously determined [18].

At first glance, the mere fact that oblate ${ }^{4} \mathrm{He}$ spinning drops have been identified $[12,16,18]$ is surprising and unexpected. Large helium drops are produced in the normal phase and may acquire angular momentum during the passage of the fluid through the nozzle of the molecular beam apparatus. The drops cool down to a temperature of $0.37 \mathrm{~K}$ [1] and are thus superfluid when they are probed. The natural question is how a spinning superfluid drop may acquire an oblate (axisymmetric) shape as it is quantum mechanically forbidden. In fact the equations characterizing the macroscopic behavior of a superfluid at zero temperature have the form of irrotational hydrodynamics, from which the moment of inertia can be calculated as $[24,35,36] \Theta_{\text {irr }}=\varepsilon^{2} \Theta_{\text {rig }}$, where

$$
\varepsilon=\frac{\left\langle y^{2}-x^{2}\right\rangle}{\left\langle y^{2}+x^{2}\right\rangle}
$$

and $\Theta_{\text {rig }}=N m\left\langle x^{2}+y^{2}\right\rangle$ is the rigid moment of inertia, with $N$ being the number of atoms in the droplet and $m$ being the atomic mass, showing that in a superfluid the value of the moment of inertia is smaller than the rigid value. In particular, for axisymmetric systems (i.e., $\left\langle x^{2}\right\rangle=\left\langle y^{2}\right\rangle$ ) the above relation gives, for the angular momentum of the superfluid, $\left\langle L_{z}\right\rangle=$ $\Theta_{\text {irr }} \omega=0$. Notice that these results are not restricted to the perturbative regime of small $\omega$ [35]. These expressions show that vortex-free oblate axisymmetric configurations cannot spin, whereas prolate (nonaxisymmetric) configurations can, and the resulting angular momentum $L_{\text {cap }}=\Theta_{\text {irr }} \omega$ is associated with the so-called capillary waves (see the discussion in the following).

Thus, the only likely explanation for the experimental observation of spinning oblate ${ }^{4} \mathrm{He}$ droplets is that these drops contain a vortex array which can store most of the angular momentum acquired during the passage through the experimental apparatus; some is taken away by the atoms emitted during the cooling process. It has, indeed, been shown $[19,20]$ that the presence of a large enough vortex array in the ${ }^{4} \mathrm{He}$ droplets confers to them a globally oblate appearance [28], similar to the case of rotating viscous droplets. Vortex arrays have been experimentally detected only in a few cases [12-14,18], as they escape detection unless drops are doped and the presence of vortices is established by the appearance of Bragg patterns from the impurities that fill the vortex cores.

The existence of helium drops that follow the sequence of prolate shapes characteristic of viscous drops under the effect of rotation is also worth discussing. In this case, axial symmetry is spontaneously broken $\left(\left\langle x^{2}\right\rangle \neq\left\langle y^{2}\right\rangle\right)$, and the droplet can store a finite amount of angular momentum in the form of capillary waves, even in the absence of vortices. In general, however, capillary waves and vortices may coexist in spinning prolate droplets, producing two quite different irrotational velocity flows compatible with the superfluidity requirement: One associated with quantized vortices and the other associated with surface capillary waves [35-38]. Remarkably, in the stability phase diagram, spinning prolate superfluid drops whose angular momentum is exclusively stored in capillary waves lie on a line [20] quite distinct from the classical line [29], as discussed in the following.

There is another unexpected difference between a rotational viscous fluid and an irrotational superfluid with finite angular momentum. When a spherical viscous droplet is set into rotation, as a consequence of centrifugal forces, it flattens out in the direction of the rotational axis: The shortest half axis of the droplet $\left(c_{z}\right.$, as defined in the following) is thus aligned with the rotational axis. However, this is not always the case for irrotational flows: Spinning vortex-free droplets with prolate shapes have, instead, the intermediate half axis $\left(b_{y}\right.$, as defined in the following) aligned with the rotational axis [33]. When vortices are present, in most cases the shortest half axis $c_{z}$ is again aligned with the rotational axis, in agreement with the classical behavior. Consequently, if only the shape of the droplet is experimentally identified, the rotational axis cannot be determined unambiguously if no further information about the angular momentum vector is available.

From the above discussion it appears that capillary waves and vortex arrays can coexist in prolate superfluid drops. This was demonstrated in a recent combined experimental and 
density functional theory (DFT) work [18]. The DFT analysis was restricted to deformable self-sustained superfluid ${ }^{4} \mathrm{He}$ cylinders which could host a fairly large vortex array due to the lower dimensionality (2D) of the computational real-space grid. This model was a generalization of the rotating, elliptic cylindric vessel filled with ${ }^{4} \mathrm{He}$ studied in Ref. [39].

In this work we complement the discussion in Ref. [18] by addressing several prolate configurations corresponding to large (for DFT standards) superfluid ${ }^{4} \mathrm{He}$ droplets: We put the emphasis on the interplay between vortices and capillary waves, we determine the equilibrium configuration for two representative values of the angular momentum, and we discuss the similarities and differences between superfluid (irrotational) and viscous (rotational) behavior. Oblate droplets hosting a different number of vortices were thoroughly studied in Ref. [19].

This paper is organized as follows: The DFT approach to liquid helium is recalled in Sec. II; Sec. III presents the results and their discussion, and a summary is given in Sec. IV. Multimedia information is presented in the Supplemental Material [40].

\section{THEORETICAL APPROACH}

Density functional theory in its static and time-dependent (TDDFT) versions has proved to be a powerful method to address superfluid helium droplets [41,42]. Within DFT, the energy of the ${ }^{4} \mathrm{He}$ droplet is written as a functional of the atom density $\rho(\mathbf{r})$ as

$$
E[\rho]=T[\rho]+E_{c}[\rho]=\frac{\hbar^{2}}{2 m} \int d \mathbf{r}|\nabla \Psi(\mathbf{r})|^{2}+\int d \mathbf{r} \mathcal{E}_{c}[\rho],
$$

where the first term is the kinetic energy, with $\rho(\mathbf{r})=|\Psi(\mathbf{r})|^{2}$, and the functional $\mathcal{E}_{c}$ contains the interaction term (in the Hartree approximation) and additional terms which describe nonlocal correlation effects [43].

The droplet equilibrium configuration is obtained by solving the Euler-Lagrange (EL) equation resulting from the functional variation of Eq. (2),

$$
\left\{-\frac{\hbar^{2}}{2 m} \nabla^{2}+\frac{\delta \mathcal{E}_{c}}{\delta \rho}\right\} \Psi(\mathbf{r}) \equiv \mathcal{H}[\rho] \Psi(\mathbf{r})=\mu \Psi(\mathbf{r}),
$$

where $\mu$ is the ${ }^{4} \mathrm{He}$ chemical potential corresponding to the number of He atoms in the droplet, $N=\int d \mathbf{r}|\Psi(\mathbf{r})|^{2}$.

To study spinning ${ }^{4} \mathrm{He}$ droplets it is convenient to work in the fixed-droplet frame of reference (corotating frame at angular velocity $\omega$ ); that is, we consider

$$
E^{\prime}[\rho]=E[\rho]-\hbar \omega\left\langle\hat{L}_{z}\right\rangle,
$$

where $\hat{L}_{z}$ is the dimensionless angular momentum operator in the $z$ direction; one looks for solutions of the EL equation resulting from the functional variation of $E^{\prime}[\rho]$,

$$
\left\{\mathcal{H}[\rho]-\hbar \omega \hat{L}_{z}\right\} \Psi(\mathbf{r})=\mu \Psi(\mathbf{r}) .
$$

The above equation has been solved using the 4HE-DFT-BCNTLS computing package [44] (see Refs. [42,45] and references therein for additional details). Briefly, we work in Cartesian coordinates, with the effective wave function $\Psi(\mathbf{r})$ defined at the nodes of a three-dimensional grid inside a calculation box large enough to accommodate the droplet in such a way that the He density is sensibly zero on the box surface. Periodic boundary conditions are imposed which allow us to use the fast Fourier transform to carry out the convolutions needed to obtain the DFT mean field $\mathcal{H}[\rho]$. The differential operators in $\mathcal{H}[\rho]$ and $\hat{L}_{z}$ are approximated by 13-point formulas, and the typical space step is $0.8 \AA$. Working at fixed angular momentum requires us to iterate on the value of $\omega$; there are efficient ways of adjusting iteratively $\omega$, e.g., the augmented Lagrangian method [46].

Angular momentum can be stored in a superfluid ${ }^{4} \mathrm{He}$ droplet in the form of surface capillary waves and/or quantized vortices $[18,20,38]$. In order to deposit angular momentum of both types in the droplet, we have used the so-called imprinting procedure by starting the imaginary-time minimization from a very flexible guess for the effective wave function $\Psi(\mathbf{r})$, namely, a superposition of a quadrupolar capillary wave and $n_{v}$ vortex lines parallel to the $z$ axis,

$$
\Psi_{0}(\mathbf{r})=\rho_{0}^{1 / 2}(\mathbf{r}) e^{\imath \alpha x y} \prod_{j=1}^{n_{v}} \frac{\left(x-x_{j}\right)+\imath\left(y-y_{j}\right)}{\sqrt{\left(x-x_{j}\right)^{2}+\left(y-y_{j}\right)^{2}}},
$$

which is then optimized by iteratively solving Eq. (5). Here, $\rho_{0}(\mathbf{r})$ is an arbitrary, vortex-free droplet density, the complex phase $e^{l \alpha x y}$ imprints a surface capillary wave with quadrupolar symmetry around the $z$ axis [24], and the product term imprints a vortex array made of $n_{v}$ linear vortices [42], where $\left(x_{j}, y_{j}\right)$ is the initial position of the $j$-vortex core. The initial value of $\alpha$ and the vortex core positions are guessed, and during the iterative solution of Eq. (5) both the vortex core structure and positions and the droplet shape change to provide, at convergence, the lowest-energy configuration.

Writing $\Psi(\mathbf{r}) \equiv \phi(\mathbf{r}) \exp [i \mathcal{S}(\mathbf{r})]$, the velocity field of the superfluid is

$$
\mathbf{v}(\mathbf{r})=\frac{\hbar}{m} \operatorname{Im}\left\{\frac{\nabla \Psi(\mathbf{r})}{\Psi(\mathbf{r})}\right\}=\frac{\hbar}{m} \nabla \mathcal{S}(\mathbf{r}) .
$$

The velocity potential $\mathcal{S}(\mathbf{r})$ gets contributions from both capillary waves and vortices in a complex way, and their contributions cannot be rigorously disentangled - the analysis needs to be model dependent [18].

The existence of different prolate configurations for a ${ }^{4} \mathrm{He}_{N}$ droplet spinning at a given $L$, each of them characterized by a number of vortices $n_{v}$, raises the question of which one is the globally stable configuration and how it depends on the number of atoms in the droplet. To determine the globally stable configuration among different ones, one has to compare their total energies $E[\rho]$, including the rotational energy (Routhian) $[29,30]$. At variance with the classical case, where a rotational energy term in the rigid-body approximation has to be added to the energy of the droplet [29,31], in the DFT approach to superfluid ${ }^{4} \mathrm{He}$ in the corotating frame this is naturally accounted for through the velocity field embodied in the phase of the effective wave function $\Psi(\mathbf{r})$ [see Eq. (7)], so one does not need to add an extra term to the total energy expression, Eq. (2), or to Eq. (5).

A difficulty inherent in the study of helium droplets is that configurations with rather distinct morphologies may have 
TABLE I. Energetics and morphology of prolate ${ }^{4} \mathrm{He}_{1500}$ droplets at $\Lambda=0.80$ for $n_{v}=0-4$. T and C indicate triangular and cross-shaped vortex arrays, respectively. The entry in boldface corresponds to the globally stable configuration. $\Lambda=0.80, L=4155, L$ cap $=4014, N=$ 1500.

\begin{tabular}{lccccc}
\hline \hline$n_{v}$ & $E(\mathrm{~K})$ & $E_{\text {kin }}(\mathrm{K})$ & $\omega(\mathrm{K} / \hbar)$ & $a_{x}(\AA)$ & $b_{y}(\AA)$ \\
\hline 0 & -7909.33 & 419.82 & 0.1145 & 42.38 & 15.93 \\
1 & -7916.16 & 478.10 & 0.1269 & 39.75 & 18.53 \\
$\mathbf{2}$ & $-\mathbf{7 9 1 8 . 8 9}$ & $\mathbf{5 3 3 . 9 6}$ & $\mathbf{0 . 1 3 4 9}$ & $\mathbf{3 6 . 5 0}$ & $\mathbf{2 0 . 7 1}$ \\
$3(\mathrm{~T})$ & -7905.11 & 594.92 & 0.1431 & 32.76 & 24.13 \\
$4(\mathrm{C})$ & -7885.24 & 635.61 & 0.1315 & 28.69 & 28.39 \\
\hline \hline
\end{tabular}

similar energies; therefore, a careful analysis is required in order to distinguish the global minimum from metastable configurations. An added challenge is that most vortex array configurations are very robust and, in spite of not being a physically conserved quantity, the starting $n_{v}$ value is often conserved instead of being relaxed to the optimal value corresponding to the global equilibrium configuration. This forces us to explore, for a given $N$-atom droplet, all possible $n_{v}$ values compatible with the chosen $L-$ a task which becomes increasingly cumbersome as $L$ increases. This robustness has, however, the benefit that one has access to a series of excited configurations, which might be experimentally accessible, characterized not only by the strictly conserved $N$ and $L$ values but also by the number of vortices $n_{v}$. We have always checked the stability of the vortex array against shape distortions.

For every stationary configuration obtained by solving Eq. (5), a "sharp density surface" is determined by calculating the locus at which the helium density equals $\rho_{0} / 2$, where $\rho_{0}$ is the atom density of the liquid; for a spherical distribution this corresponds to the sphere of radius $R$ defined below. In the case of deformed droplets, three lengths (half axes), $a_{x}, b_{y}$, and $c_{z}$, are introduced, representing the distances from the center of mass of the droplet to the sharp surface along the principal axes of inertia. These lengths are represented in the inset in Fig. 1. For an axisymmetric droplet, $a_{x}=b_{y} \neq c_{z}$. These lengths have been used to characterize the droplet shape by defining two dimensionless aspect ratios, $a_{x} / c_{z}$ and $b_{y}^{3} / V$ [16,20,32], where $V$ is the volume of the nonrotating spherical droplet.

The shapes of classical drops exclusively subjected to surface tension and centrifugal forces follow a universal stability diagram in terms of the dimensionless angular momentum $\Lambda$ and angular velocity $\Omega$, defined by $[29,31]$

$$
\begin{aligned}
& \Omega \equiv \sqrt{\frac{m \rho_{0} R^{3}}{8 \gamma} \omega,} \\
& \Lambda \equiv \frac{\hbar}{\sqrt{8 \gamma R^{7} m \rho_{0}}} L,
\end{aligned}
$$

where $m$ is the atom mass and $\gamma$ is the surface tension of the liquid. For liquid ${ }^{4} \mathrm{He}$ at zero temperature and pressure, $\gamma=0.274 \mathrm{~K}^{-2}$, and $\rho_{0}=0.0218 \AA^{-3}$, the sharp radius of the spherical droplet is given by $R=r_{0} N^{1 / 3}$, with $r_{0}=2.22 \AA$ [41], and $\hbar^{2} / m=12.12 \mathrm{~K}^{2}$. We recall that $\hbar=7.6382 \mathrm{~K} \mathrm{ps}$ and that $\hbar c=2.2899 \times 10^{7} \mathrm{~K} \AA$.

We want to stress that this universality is lost if one goes beyond that simple model, e.g., by using a DFT description of the liquid, since this incorporates effects such as surface diffuseness and liquid incompressibility [47]: Although such effects are expected to be less important as the droplet size increases, they can be tangible for the droplet sizes investigated here. Yet Eqs. (8) are very useful as they allow for a comparison between experimental and calculated droplets, even if the latter are much smaller (for obvious computational reasons) than those probed in the experiments. However, one should have in mind that some differences may appear in the comparison due to these unavoidable finite-size effects. In addition, the presence of vortices in the case of superfluid droplets definitely breaks the universality of the stability diagram, as shown below.

\section{RESULTS AND DISCUSSION}

Systematic DFT calculations are very cumbersome to carry out, especially when several configurations hosting different

TABLE II. Energetics and morphology of prolate ${ }^{4} \mathrm{He}$ droplets with $N=20000$ at $\Lambda=0.80$ for $n_{v}=0-5$. L, C, and P indicate linear, cross-shaped, and pentagonal vortex arrays, respectively. The entry in boldface corresponds to the globally stable configuration, and the asterisk identifies the highest-energy $n_{v}=4$ configuration. $\Lambda=0.80, L=85317, L_{\text {cap }}=82449, N=20000$.

\begin{tabular}{lcccccc}
\hline \hline$n_{v}$ & $E(\mathrm{~K})$ & $E_{\text {kin }}(\mathrm{K})$ & $\omega(\mathrm{K} / \hbar)$ & $a_{x}(\AA)$ & $b_{y}(\AA)$ & $c_{z}(\AA)$ \\
\hline 0 & -127236.24 & 2319.50 & 0.03172 & 100.07 & 37.75 & 44.90 \\
1 & -127320.36 & 2495.08 & 0.03422 & 96.22 & 41.65 & 44.75 \\
2 & -127397.02 & 2654.06 & 0.03607 & 91.70 & 44.51 & 45.97 \\
$\mathbf{3}(\mathbf{L})$ & $-\mathbf{1 2 7 4 3 2 . 2 8}$ & $\mathbf{2 8 3 1 . 7 8}$ & $\mathbf{0 . 0 3 7 3 3}$ & $\mathbf{8 7 . 1 1}$ & $\mathbf{4 7 . 8 8}$ & $\mathbf{4 4 . 7 3}$ \\
$4(\mathrm{C})$ & -127428.73 & 3021.48 & 0.03908 & 82.20 & 52.50 & 46.62 \\
$4^{*}(\mathrm{~L})$ & -127405.10 & 3027.91 & 0.03745 & 82.85 & 51.37 & 46.43 \\
$5(\mathrm{P})$ & -127413.79 & 3214.34 & 0.04021 & 75.66 & 58.05 & 47.34 \\
\hline \hline
\end{tabular}


TABLE III. Energetics and morphology of prolate ${ }^{4} \mathrm{He}$ droplets with $N=35000$ at $\Lambda=0.80$ for $n_{v}=0-6$. L, C, and P indicate linear, cross-shaped, and pentagonal vortex arrays, respectively. The $6(\mathrm{Ct})$ configuration has a center vortex, i.e., along the rotational axis. The entry in boldface corresponds to the globally stable configuration, and an asterisk identifies the highest-energy configuration among those with the same $n_{v}$ value; in particular, $6^{*}$ corresponds to a six-vortex array configuration in which the six vortices are at the vertices of a hexagon. $\Lambda=0.80, L=163899, L_{\text {cap }}=158398, N=35000$.

\begin{tabular}{|c|c|c|c|c|c|c|}
\hline$n_{v}$ & $E(\mathrm{~K})$ & $E_{\text {kin }}(\mathrm{K})$ & $\omega(\mathrm{K} / \hbar)$ & $a_{x}(\AA)$ & $b_{y}(\AA)$ & $c_{z}(\AA)$ \\
\hline 0 & -227393.50 & 3360.11 & 0.02402 & 120.49 & 45.42 & 54.21 \\
\hline 1 & -227522.50 & 3581.02 & 0.02580 & 116.29 & 49.68 & 54.09 \\
\hline 3 & -227698.13 & 3986.17 & 0.02789 & 106.56 & 56.28 & 53.89 \\
\hline $4(C)$ & -227721.40 & 4233.29 & 0.02927 & 101.38 & 61.12 & 56.03 \\
\hline $4^{*}(\mathrm{~L})$ & -227700.04 & 4233.98 & 0.02829 & 101.76 & 60.15 & 55.82 \\
\hline $6(\mathrm{Ct})$ & -227699.01 & 4732.32 & 0.03130 & 85.12 & 75.48 & 53.36 \\
\hline $6^{*}$ & -227697.29 & 4710.33 & 0.03012 & 87.06 & 73.60 & 57.59 \\
\hline
\end{tabular}

numbers of vortices have to be analyzed and their geometries have to be tested against distortions of the vortex array. This hinders a systematic exploration of the phase diagram. For this reason, we have limited our study to some relevant cases which embody the physics we aim to discuss. We have focused in particular on two $\Lambda$ values that embrace a fairly large range of prolate configurations [20], namely, $\Lambda=0.80$ and 1.25, for droplets with $N=1500$ atoms (a value for which a rather detailed series of DFT calculations is available [20]) and with $N=20000$ and 35000 ; these large prolate droplets (for DFT standards) are well suited to disclose the interplay between capillary waves and vortex arrays, the object of this study, and how the number of vortices in the globally stable configuration evolves as a function of $N$.

Tables I-VI collect the results obtained for the mentioned $(\Lambda, N)$ values (one pair of values per table) and give details on their energetics and morphology. In each table we highlight in boldface the entry corresponding to the globally stable configuration, i.e., that with the lowest energy for the given values of $N$ and $\Lambda$. When more than one configuration has been found for a given number of vortices $n_{v}$, the more energetic one is identified with an asterisk $\left(^{*}\right)$. Triangular, cross-shaped, pentagonal, and linear vortex arrays are denoted by T, C, P, and $\mathrm{L}$, respectively. Notice that the configurations in a given table can be directly compared, as they correspond to the same values of two strictly conserved quantities in isolated droplets.

As already discussed in the Introduction, it appears from Tables I-VI that for vortex-free superfluid droplets the intermediate half axis $b_{y}$ is aligned with the rotational axis, at variance with the classical rotating droplets, for which the shortest half axis $c_{z}$ is aligned instead with the rotational axis. We are not aware of a general demonstration of this counterintuitive result; its proof is, however, known for ellipsoidal drops made of irrotational (potential) fluids [33]. This peculiarity of spinning irrotational drops has to be taken into account for a proper analysis of the experiments: Identifying the shortest axis of a spinning superfluid ${ }^{4} \mathrm{He}$ droplet with the rotational axis might be incorrect. For vortex-hosting superfluid droplets, though, the rotational axis coincides most of the time with the shortest $\left(c_{z}\right)$ one, as for viscous drops. But there are exceptions, e.g., for $N=20000$ and 35000 when $n_{v}=2$.

In most cases, the presence of a vortex array confers to the droplet the appearance of a rotating viscous droplet. A notorious example of this apparently classical behavior is the meniscus that develops at the liquid-vapor interface in a rotating bucket filled with superfluid helium above the critical angular velocity required for vortex nucleation $[37,48]$. Hence, determining the angular momentum of the droplet (magnitude and direction) and whether it hosts a vortex array or not seems unavoidable before drawing a definite conclusion about how superfluid helium droplets rotate.

We show in Fig. 1 the dimensionless ratios $b_{y}^{3} / V$ versus $a_{x} / c_{z}$ for the configurations collected in Tables I-VI, irrespective of whether they correspond to globally stable or metastable configurations. The $N=1500$ results are complemented with those shown in Fig. 1 of Ref. [20], which presents data for a large sample of $\Lambda$ values. Also shown are the experimental results [16], which have an average atom

TABLE IV. Energetics and morphology of prolate ${ }^{4} \mathrm{He}_{1500}$ droplets at $\Lambda=1.25$ for $n_{v}=0-4$. $L$ indicates a linear vortex array. The entry in boldface corresponds to the globally stable configuration. $\Lambda=1.25, L=6493, L_{\text {cap }}=6224, N=1500$.

\begin{tabular}{lccccr}
\hline \hline$n_{v}$ & $E(\mathrm{~K})$ & $E_{\text {kin }}(\mathrm{K})$ & $\omega(\mathrm{K} / \hbar)$ & $a_{x}(\AA)$ & $b_{y}(\AA)$ \\
\hline $\mathbf{0}$ & $\mathbf{- 7 6 5 8 . 1 0}$ & $\mathbf{5 1 9 . 1 1}$ & $\mathbf{0 . 1 0 0 1}$ & $\mathbf{4 8 . 4 1}$ & $\mathbf{1 3 . 6 3}$ \\
1 & -7641.16 & 583.36 & 0.1025 & 46.68 & 15.80 \\
2 & -7626.09 & 648.30 & 0.1153 & 44.44 & 17.15 \\
$3(\mathrm{~L})$ & -7601.71 & 714.49 & 0.1197 & 42.51 & 18.72 \\
$4(\mathrm{~L})$ & -7567.76 & 763.02 & 0.1222 & 42.13 & 16.52 \\
\hline \hline
\end{tabular}


TABLE V. Energetics and morphology of prolate ${ }^{4} \mathrm{He}$ droplets with $N=20000$ at $\Lambda=1.25$ for $n_{v}=0-5$. L and C indicate linear crossshaped vortex arrays, respectively. The entry in boldface corresponds to the globally stable configuration, and the asterisk identifies the highestenergy $n_{v}=4$ configuration. $\Lambda=1.25, L=133307, L_{\text {cap }}=127937, N=20000$.

\begin{tabular}{|c|c|c|c|c|c|c|}
\hline$n_{v}$ & $E(\mathrm{~K})$ & $E_{\text {kin }}(\mathrm{K})$ & $\omega(\mathrm{K} / \hbar)$ & $a_{x}(\AA)$ & $b_{y}(\AA)$ & $c_{z}(\AA)$ \\
\hline $\mathbf{0}$ & -125806.09 & 2883.61 & 0.02781 & 114.26 & 32.39 & 36.54 \\
\hline 1 & -125789.56 & 3097.10 & 0.02953 & 111.73 & 35.85 & 36.16 \\
\hline 3 & -125767.52 & 3494.95 & 0.03203 & 105.28 & 39.80 & 38.28 \\
\hline $4^{*}(\mathrm{C})$ & -125701.35 & 3676.09 & 0.03306 & 103.20 & 42.47 & 38.92 \\
\hline $4(\mathrm{~L})$ & -125714.08 & 3705.57 & 0.03283 & 102.53 & 41.98 & 39.20 \\
\hline
\end{tabular}

number $\langle N\rangle=6 \times 10^{9}$, and the result for classical rotating drops [32]. While the results for vortex-hosting configurations are similarly distributed and roughly follow the classical drops trend, Fig. 1 shows that vortex-free prolate configurations form a separate branch (capillary branch) displaying a completely different behavior. Both branches meet at the $\left(a_{x} / c_{z}, b_{y}^{3} / V\right)=(1,3 / 4 \pi)$ point corresponding to spherical droplets.

It is worth noticing that configurations with the same $n_{v}$ but rather different $N$ values yield similar points on the $\left(a_{x} / c_{z}, b_{y}^{3} / V\right)$ plane, especially when the vortex array has the same morphology (linear, cross, etc.), in particular for vortexfree configurations. The universality of the classical results, which allows us to scale them with $N$, is not completely lost. Unfortunately, even $N=35000$ is too small a value compared to the experimental ones to allow us to draw any sensible conclusion about this issue. Figure 1 also shows that, for a fixed $\Lambda$ value, the larger $n_{v}$ is, the more similar viscous and vortex-hosting droplets are.

We thus see that knowledge of the shape of a large number of deformed configurations is not enough to unambiguously characterize their rotational behavior as classical or superfluid and that one has to combine this information with the simultaneous determination of the angular momentum of the droplet by, e.g., obtaining the stability diagram in the $(\Lambda, \Omega)$ plane. This diagram is shown in Fig. 2 for some of the configurations in Fig. 1. While the capillary branch may arrive up to nearly $\Lambda=0$, as only a small deformation is needed for the droplet to sustain capillary waves, the vortex-hosting branch must abruptly interrupt at some $\left(\Lambda_{c}, \Omega_{c}\right)$ point, as a critical angular velocity $\omega_{c}$ is needed for nucleating a vortex line in a superfluid droplet [36]. The red symbols in Fig. 2 represent the critical vortex nucleation point for the three $N$ values used in this study. For the sake of completeness, we also display the DFT result for $N=5000$ [26] and that of the hollow-core model [49] for an $N=10^{9}$ drop taking for the vortex core radius a value of $1 \AA$.

The existence of this critical point dramatically distinguishes superfluid from viscous droplet rotational behavior, as no axisymmetric superfluid $\mathrm{He}$ droplet can be set into rotation for $\Lambda<\Lambda_{c}$. Another conspicuous difference between viscous and superfluid vortex-hosting droplet results can be observed in Fig. 2, namely, the location of the oblate-to-prolate bifurcation point. For viscous droplets, it is at $\Lambda=1.2$ [30,31], whereas for droplets of the size studied here, DFT yields a bifurcation slightly below $\Lambda=0.8$. Indeed, the three-vortex ${ }^{4} \mathrm{He}_{1500}$ configurations connected by a red horizontal line correspond to oblate (left) and prolate (right) configurations. We attribute this large difference to a finite-size effect, although we cannot ascertain this. In Fig. 2, finite-size effects also clearly affect the position of the critical vortex nucleation point.

Comparing the results for the same $N$ value, one can see from Tables I-VI that the ratio $b_{y} / a_{x}$ decreases as $\Lambda$ increases. This causes, e.g., for the ${ }^{4} \mathrm{He}_{1500}$ droplet, the most stable $n_{v}=4$ vortex array, which was cross shaped at $\Lambda=0.80$,

TABLE VI. Energetics and morphology of prolate ${ }^{4} \mathrm{He}$ droplets with $N=35000$ at $\Lambda=1.25$ for $n_{v}=0-6$. L, C, and P indicate linear, cross-shaped, and pentagonal vortex arrays, respectively. The six-vortex array configuration is a stretched hexagon. The entry in boldface corresponds to the globally stable configuration, and an asterisk identifies the highest-energy configuration among those with the same $n_{v}$ value. $\Lambda=1.25, L=256093, L_{\text {cap }}=245756, N=35000$.

\begin{tabular}{|c|c|c|c|c|c|c|}
\hline$n_{v}$ & $E(\mathrm{~K})$ & $E_{\text {kin }}(\mathrm{K})$ & $\omega(\mathrm{K} / \hbar)$ & $a_{x}(\AA)$ & $b_{y}(\AA)$ & $c_{z}(\AA)$ \\
\hline 1 & -225307.23 & 4450.00 & 0.02225 & 134.88 & 42.80 & 43.50 \\
\hline 3 & -225312.04 & 4950.83 & 0.02402 & 127.81 & 47.07 & 46.14 \\
\hline $4^{*}(\mathrm{C})$ & -225239.14 & 5198.51 & 0.02477 & 125.33 & 50.06 & 46.70 \\
\hline $4(\mathrm{~L})$ & -225263.35 & 5217.47 & 0.02464 & 124.69 & 49.46 & 47.12 \\
\hline 6 & -225104.71 & 5713.58 & 0.02600 & 118.84 & 54.80 & 48.43 \\
\hline
\end{tabular}




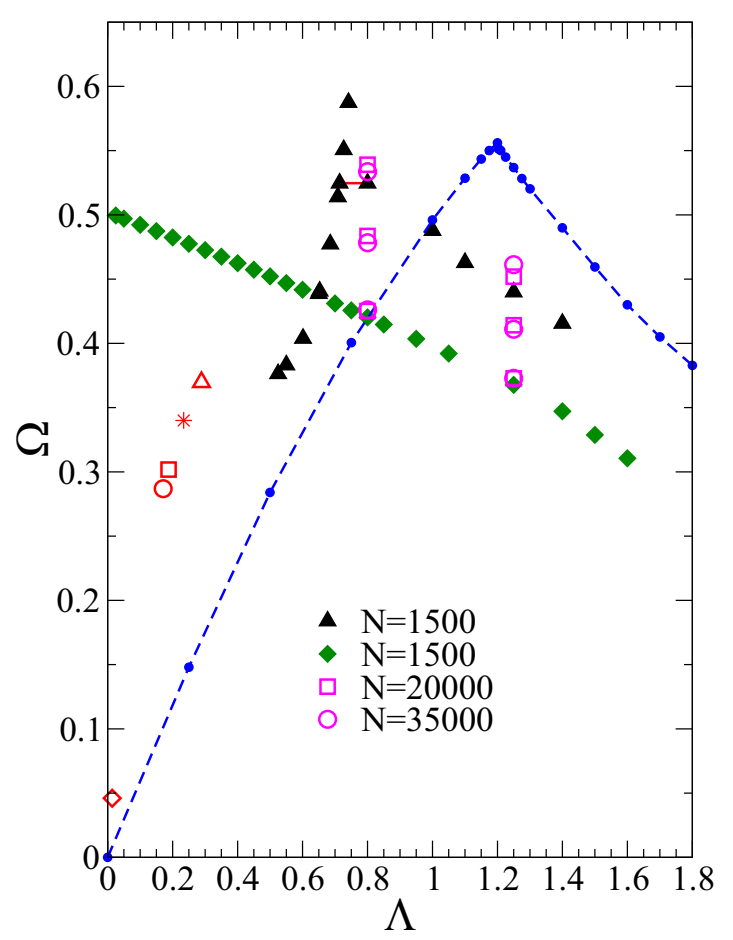

FIG. 2. Dimensionless angular velocity $\Omega$ vs dimensionless angular momentum $\Lambda$. Black triangles: Three-vortex ${ }^{4} \mathrm{He}_{1500}$ configurations [20]. Green diamonds: Vortex-free (capillary wave) ${ }^{4} \mathrm{He}_{1500}$ configurations [20]. The oblate and prolate configurations connected by a red horizontal line at $\Omega=0.5247$ are the twin configurations we refer to in the text. Magenta squares (circles) represent the $N=$ 20000 (35000) results for $n_{v}=0,2$, and 5 configurations (for given $N$ and $\Lambda$ values, the larger $n_{v}$ is, the bigger $\Omega$ is). For the sake of completeness, we also include the critical points for nucleating a single vortex (red symbols): Triangle, $N=1500$; asterisk, $N=5000$ [26]; square, $N=20000$; circle, $N=35000$. The red diamond at $(\Lambda, \Omega)=\left(1.4 \times 10^{-2}, 4.6 \times 10^{-2}\right)$ is the critical point for nucleating a vortex for $N=10^{9}$ in the hollow core model. The blue dots connected by a dashed line are the classical rotating drop results $[31,32]$.

to become instead linear at $\Lambda=1.25$; the same happens for $N=20000$. Large droplets can host both kinds of vortex arrangements (see, e.g., Table VI). For a given $\Lambda$, a large droplet may accommodate a nonlinear vortex array more easily. Notice, for instance, that, at $\Lambda=1.25$, the cross-shaped $n_{v}=4$ configuration for $N=35000$ is still more stable than the linear one. The existence of multiple configurations for the same $n_{v}$ value is also ubiquitous in axisymmetric configurations [19,50,51], making the search for the lowest-energy configuration very time-consuming.

Further inspection of Tables I-VI shows that, for given $N$ and $\Lambda$ values, the globally stable spinning prolate configurations are not necessarily vortex free and that, contrary to a naive expectation, for large, fixed $N$ and $\Lambda$ values, the energy is not monotonously varying with $n_{v}$; see, for instance, Tables I, II, and VI. We have found that for the largest $\Lambda$ value considered in this study $(\Lambda=1.25)$, the vortex-hosting configuration becomes the globally stable configuration only above a "critical" $N$ value. We have checked that for $N=30000$ the vortex-free and the $n_{v}=2$
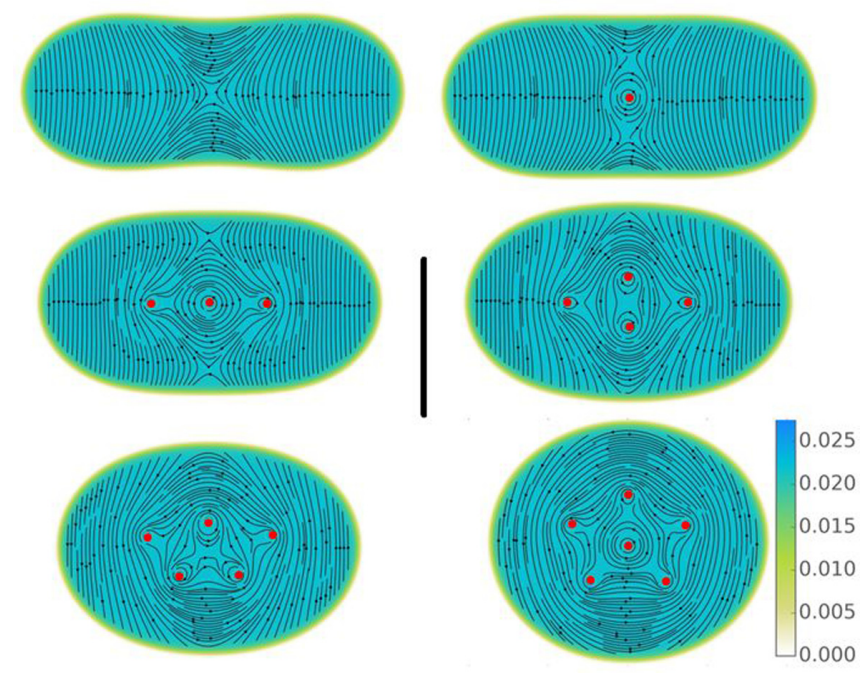

FIG. 3. Densities on the symmetry plane perpendicular to the rotational axis for the $N=35000 \mathrm{He}$ droplet hosting zero, one, and $n_{v}=3-6$ vortices at $\Lambda=0.80$. The streamlines of the superfluid flow are superimposed, and their direction is counterclockwise. The positions of the vortex cores are marked by red dots for visualization. The color bar shows the atom density (in $\AA^{-3}$ ), and the black bar represents a distance of $100 \AA$.

configurations are nearly degenerate with energies of $-191994.86 \mathrm{~K}$ and $-191994.49 \mathrm{~K}$, respectively. As Table VI shows, the globally stable configuration of the $N=35000$ droplet is the $n_{v}=2$ one. It is thus very likely that the prolate large $N$ drops studied in the FEL [12] and XUV [16] experiments contain both vortex arrays and capillary waves [18]. Notice also that the energy differences between the first excited configurations and the globally stable ones are small, and it cannot be completely discarded that some such excited configurations are also detected in the experiments. These considerations indicate that vortex-free prolate configurations might be extremely difficult to detect either because they correspond to globally stable configurations only for small- $N$ droplets that escape detection in current diffractive imaging experiments or because they are metastable configurations in the case of large- $N$ drops and likely decay to vortex-hosting configurations before they are probed.

Figures 3 and 4 show some $2 \mathrm{D}$ densities corresponding to the $N=35000$ droplet. One may see that, in order to store a large angular momentum, a vortex-free configuration has to be very stretched; see the discussion on capillary waves below. This is a general trend that implies an increase of the droplet surface energy; as shown in Tables I-VI, the kinetic energy also increases with the number of vortices. The globally stable configuration is determined by a delicate balance between these two competing effects. Figure 4 shows that the $N=35000$ droplet is large enough to accommodate up to six vortices and that the shape of the droplet evolves from two lobed to a more compact, "pill-shaped" appearance as found in the experiments [14,16,18]. Although most of these configurations are metastable, these shape transitions exemplify the competition between compact and more linear vortex array arrangements to determine the appearance of the 

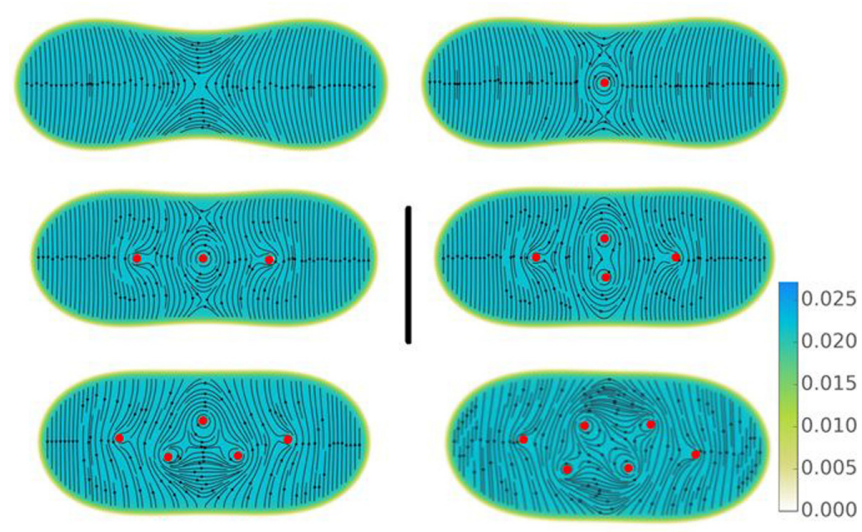

FIG. 4. Densities on the symmetry plane perpendicular to the rotational axis for the $N=35000 \mathrm{He}$ droplet hosting zero, one, and $n_{v}=3-6$ vortices at $\Lambda=1.25$. The streamlines of the superfluid flow are superimposed, and their direction is counterclockwise. The positions of the vortex cores are marked by red dots for visualization. The color bar shows the atom density (in $\AA^{-3}$ ), and the black bar represents a distance of $100 \AA$.

droplet. Notice that Fig. 1 also displays the same effect: As $n_{v}$ increases for a given $\Lambda$, the point representing the vortexhosting configuration dramatically displaces to smaller $a_{x} / c_{z}$ values, yielding at the same time a larger $b_{y}^{3} / V$ and hence more compact droplet shapes. It is also worth seeing from the 2D figures that two-lobed configurations such as those reported in Refs. [14,16] appear when $n_{v}$ is small; increasing $n_{v}$ increases the neck radius between the lobes, as the vortex array becomes less linear and more similar to a patch of a triangular arrangement (as expected when $n_{v}$ becomes very large). Since the number of pill-shaped droplets found in the experiment [16] is six times larger than that of dumbbell-like droplets, this would again indicate that large, very prolate droplets host fairly large vortex arrays.

Figure 5 displays a side view of the $N=35000$ droplet at $\Lambda=1.25$ for the $n_{v}=0-3$ configurations. One can see how vortices locally distort the droplet surface as they have to hit it perpendicularly. A similar figure can be found in Ref. [19] for oblate configurations. Thus, droplets hosting large vortex arrays are far from being ellipsoidal. In the diffractive imaging experiments, the images used to identify the presence of vortices with a well-oriented direction of their cores [18] would

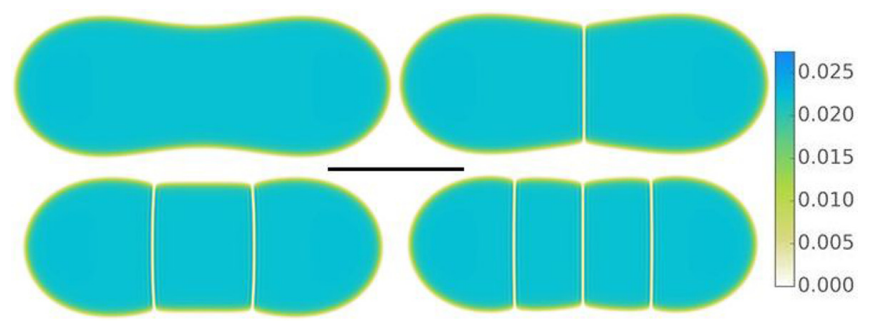

FIG. 5. Densities on the symmetry plane containing the vortex lines for the $N=35000$ droplet hosting $n_{v}=0-3$ vortices at $\Lambda=$ 1.25. The color bar shows the atom density (in $\AA^{-3}$ ), and the black bar represents a distance of $100 \AA$. correspond to the flat surfaces limited from above and below the droplet side view shown Fig. 5.

Vortex arrays in prolate configurations are distorted, as found in the experiments [18] and displayed in the 2D figures. To characterize these distortions, we have calculated an oblate configuration "twin" of the prolate $N=1500, \Lambda=0.80$, $n_{v}=3$ configuration for the same value of $\Omega$, namely, 0.5247 , which implies a lower $\Lambda$ value (see Fig. 2). For this $n_{v}=3$ oblate configuration we have found $\Lambda=0.714, a_{x} / c_{z}=1.38$, and $b_{y}^{3} / V=0.318$; the latter two values should be compared to those of its twin prolate configuration, $a_{x} / c_{z}=1.68$ and $b_{y}^{3} / V=0.207$. In the oblate $n_{v}=3$ configuration the vortex array forms an equilateral triangle with an intervortex distance of $16.6 \AA$. In the $n_{v}=3$ prolate configuration the triangle is isosceles and stretched in the direction of the largest principal axis of inertia ( $x$ axis). The stretched intervortex distance is $22.1 \AA$, and the other two sides of the triangle correspond to an intervortex distance of $15.4 \AA$.

All configurations displayed in the $2 \mathrm{D}$ figures are stationary in the corotating frame; as a consequence, they would be seen from the laboratory frame as if they were rotating, apparently like a rigid body, with the angular frequency $\omega$ imposed to obtain them. Let us recall and stress that this is a misleading appearance. The motion of a fluid is a combination of translation, rotation, and deformation of the fluid elements, and only when the vorticity (defined as the curl of the velocity field of the fluid) is nonzero may one speak of a true rotation, with distinguishing between the rotation and motion of the fluid elements along a curved path [37,52], e.g., around a vortex core, being important. Since the velocity field of the superfluid is potential, vorticity is zero except on the vortex lines; in their absence, vorticity is zero everywhere.

To illustrate this situation, we have simulated within the TDDFT approach [42] the time evolution of the prolate ${ }^{4} \mathrm{He}_{1500}$ droplet for the vortex-free and $n_{v}=3$ configurations at $\Lambda=0.8$. Figure 6 shows, for the $n_{v}=3$ case, the trajectory $\left(x_{c}, y_{c}\right)$ of the vortex cores on the $z=0$ plane. It can be observed that the intervortex distances do not change during the evolution, indicating that the three-vortex array rotates with the angular velocity imposed on the corotating frame, $\omega=1.871 \times 10^{-2} \mathrm{ps}^{-1}$. We see this dynamics as the rigid rotation of the vortex cores (which is not in contradiction with the previous discussion, as they are empty), around which the irrotational superflow accommodates in its displacement. One may also notice the accuracy of the stationary solution we have obtained; otherwise, the vortex core trajectories, plotted here point by point, would not be the perfect circumferences displayed in Fig. 6. Figure 6 also exemplifies the robustness of the fixed- $n_{v}$ configurations mentioned in Sec. II. These configurations-which are stationary in the corotating frame-likely correspond to local energy minima and are separated by an energy barrier from other configurations with a different $n_{v}$ but the same $N$ and $\Lambda$ values.

The interested reader will find two videos in the Supplemental Material [40] showing the time evolution of these two configurations in the corotating frame for about $450 \mathrm{ps}$. It may be illuminating to look at the time evolution of the streamlines pattern: It shows how different the apparent rotation of the superfluid droplet is, compared to the true rotation of a viscous drop. We want to stress that the energy, particle number, and 


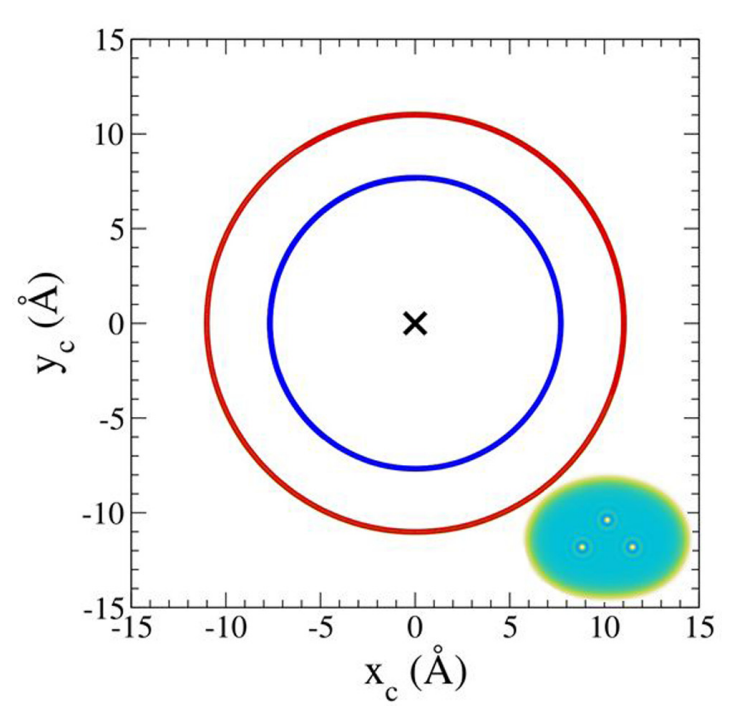

FIG. 6. Trajectories of the vortex cores $\left(x_{c}, y_{c}\right)$ on the $z=0$ plane corresponding to the prolate $N=1500, \Lambda=0.80, n_{v}=3$ configuration. One vortex is turning around the center of mass of the droplet (shown by a cross) at a distance of $7.7 \AA$, while the other two vortices rotate at a distance of $11.0 \AA$. The inset represents the droplet density on the symmetry plane perpendicular to the rotational axis.

angular momentum of the isolated droplet are strictly conserved during the evolution.

It is worth recalling that vorticity in quantum fluids may often lead to a turbulent regime [53,54] like that resulting from the decay of vortex tangles in bulk liquid ${ }^{4} \mathrm{He}$ or the turbulence in Bose-Einstein condensates (BECs) generated by quickly stirring the condensate using laser beams. Our vortex structures, however, are stable against decay, reconnections, or other mechanisms which are known to lead to turbulent behavior in liquid ${ }^{4} \mathrm{He}$ or BECs. This is what we, indeed, observe in our simulations: In spite of the possibility of bending and displacing the vortex cores, which is allowed by the three-dimensional nature of our calculations, either the vortex structures we find are stationary states in the corotating system, or when allowed to evolve under real-time dynamics, they rotate around the central axis but remain otherwise stable, without showing any tendency to be expelled or reconnect with nearby vortices. This is in agreement with the experimental measurements on nanoscopic ${ }^{4} \mathrm{He}$ droplets hosting vortices in which no sign of decay of the vortex structures is observed [18]. Turbulent behavior may arise, however, in nanoscopic ${ }^{4} \mathrm{He}$ droplets under special conditions, as illustrated in Ref. [55], where a TDDFT study of the merging of two vortex-free superfluid ${ }^{4} \mathrm{He}$ droplets showed that vortex rings are dynamically nucleated during the merging process and that their annihilation produces a massive emission of rotons and subsequent turbulent behavior, whose scaling follows the very general power laws underlying turbulence in quantum fluid systems [53,54].

Streamlines of the superfluid flow are shown in Figs. 3 and 4. The superflow follows the direction of the angular frequency imposed on the corotating frame (counterclockwise in this case). Streamlines allow us to infer by visual inspection the coexistence of vortices and surface capillary waves, as their velocity fields are very different. The streamlines associated with vortices wrap around their cores, whereas those associated with capillary waves end abruptly at the droplet surface [18,24,39]. As already mentioned, separating the contribution of capillary waves and vortices in the velocity fields is generally not possible, with the superfluid velocity field being proportional to the gradient of the phase $\mathcal{S}(\mathbf{r})$ of the effective wave function $\Psi(\mathbf{r})$, where the contributions of both vortices and capillary waves are entangled.

The coexistence of one single vortex and a quadrupolar irrotational flow in an elliptic cylinder filled with helium rotating at constant $\omega$ was studied by Fetter [39], who anticipated the possible appearance of a row of vortices along the longer axis of the elliptic vessel cross section. At variance with isolated droplets, the cylinder has a rigid surface of known geometry. Writing the phase of $\Psi(\mathbf{r})$ as the sum of a term arising from the vortex line and another from the quadrupolar flow [the same guess that we made in Eq. (6), but in our case only for the starting configuration], Fetter could determine how the angular momentum is shared between the vortex and the quadrupolar irrotational flow. It does not look feasible to extend the cylinder approach to the $n_{v}>1$ case. Even for one single vortex, the study of isolated droplets is much more complex, as their shapes are not ellipsoidal in general and they are known only after the stationary configuration has been determined by solving Eq. (5).

Yet to analyze the experimental results [18] it has been useful to know, at least approximately, how angular momentum is shared between vortices and capillary waves. In order to do that, we computed the deformation parameter $\varepsilon$ defined in Eq. (1) using the $a_{x}$ and $b_{y}$ parameters given in Tables I-VI for the $n_{v}=0$ configurations and the relation giving the angular momentum associated with capillary waves, $L_{\text {cap }}=\varepsilon^{2} \Theta_{\text {rig }} \omega$ [see the discussion following Eq. (1)].

We have found that it works surprisingly well, as it coincides with the exact $L$ calculated within the DFT approach to within a few percent. The $L_{\text {cap }}$ value obtained for each studied $n_{v}=0$ configuration is given in Tables I-VI. For vortex-hosting configurations, the angular momentum stored in the vortex arrays may then be estimated by subtracting $L_{\text {cap }}$ from the total $L$. The validity of this procedure cannot be assessed, however. So far, the only possibility to experimentally determine the angular momentum of the drop has been to use Feynman's formula [56] to obtain the angular velocity $\omega$ from the vortex density, which could be determined from the 2D droplet images and the above expression for $L_{\text {cap }}$ (see Ref. [18] for details).

\section{SUMMARY}

We have shown that the presence of vortex arrays in spinning superfluid ${ }^{4} \mathrm{He}$ droplets and their interplay with capillary waves have a profound influence on the determination of their globally stable configuration. The presence of vortex arrays, irrespective of whether they are detected or not [20], is the only plausible explanation for the existence of oblate configurations, which otherwise would be in conflict with quantum mechanics or would imply that droplets are not superfluid, 
which is extremely unlikely due to the working temperatures in ${ }^{4} \mathrm{He}$ droplet experiments [1].

Surface capillary waves are ubiquitous in prolate superfluid ${ }^{4} \mathrm{He}$ droplets. Depending on their size and angular momentum, these waves may coexist with vortex arrays. We have found that the global equilibrium configuration of small prolate droplets is vortex free, but the situation changes as the droplet size increases. This result is in agreement with a recent experiment [18], which disclosed that vortex arrays and capillary waves coexist in very large drops.

While vortex arrays may be detected by doping the droplets, capillary waves are very elusive and escape direct detection. This poses a serious difficulty to determine the angular momentum of the superfluid droplet. From the theoretical side, microscopic approaches such as DFT treat vortex arrays and capillary waves on the same footing, and one has to resort to approximate methods to disentangle their contributions to the total angular momentum of the droplet. Determining, even in a model-dependent way, the angular momentum of the droplets is essential if one wants to characterize spinning superfluid droplets, as only simultaneous knowledge of the droplet morphology and angular momentum allows for a sensible comparison with classical or quantum models.

In contrast to viscous droplets executing rigid-body rotation (modeled by viscous droplets subject to surface tension and centrifugal forces alone), the stability phase diagram of superfluid droplets is not universal and cannot be characterized by dimensionless angular momentum $\Lambda$ and dimensionless angular velocity $\Omega$ as its classical counterpart. Their knowledge does not determine univocally the rotational properties of superfluid helium droplets, which display a clear dependence on the droplet size and/or the number of vortices they host. This is not in contradiction to the recent finding [18] that big superfluid ${ }^{4} \mathrm{He}$ droplets hosting a large number of vortices seem to rotate like rigid bodies, thus following the classical stability phase diagram. Rather, it is a manifestation of finite-size effects which still have to be studied in detail and, together with recent works on rotating ${ }^{3} \mathrm{He}$ droplets [47,57] and mixed ${ }^{3} \mathrm{He}-{ }^{4} \mathrm{He}$ droplets [58,59], call for further experimental research.

\section{ACKNOWLEDGMENTS}

We are indebted to A. Vilesov for useful discussions. We thank T. Möller and B. Langbehn for providing us with the results of Ref. [16] shown in Fig. 1 and S. Butler for providing us with the results of the classical model calculations used in this work. This work has been performed under Grant No. FIS2017-87801-P (AEI/FEDER, UE). J.M.E. acknowledges support from the Spanish Research Agency (AEI) through the Severo Ochoa Centres of Excellence program (Grant No. SEV-2017-0706) and from the European Union MaX Center of Excellence (EU-H2020 Grant No. 824143).
[1] J. P. Toennies and A. F. Vilesov, Angew. Chem. Phys. 43, 2622 (2004).

[2] R. M. P. Tanyag, C. F. Jones, C. Bernardo, S. M. O. O'Connell, D. Verma, and A. F. Vilesov, in Cold Chemistry: Molecular Scattering and Reactivity Near Absolute Zero, edited by $\mathrm{O}$. Dulieu and A. Osterwalder (The Royal Society of Chemistry, London, 2018).

[3] K. K. Lehmann and G. Scoles, Science 279, 2065 (1998).

[4] C. Callegari and W. E. Ernst, in Handbook of High Resolution Spectroscopy (Wiley, Hoboken, NJ, 2011), Vol 3, p. 1551.

[5] Ph. Sindzingre, M. L. Klein, and D. M. Ceperley, Phys. Rev. Lett. 63, 1601 (1989).

[6] M. V. Rama Krishna and K. B. Whaley, Phys. Rev. Lett. 64, 1126 (1990).

[7] S. Grebenev, J. P. Toennies, and A. F. Vilesov, Science 279, 2083 (1998).

[8] E. Krotscheck and R. Zillich, J. Chem Phys. 115, 10161 (2001).

[9] J. Tang, Y. Xu, A. R. W. McKellar, and W. Jäger, Science 297, 2030 (2002).

[10] N. B. Brauer, S. Smolarek, E. Loginov, D. Mateo, A. Hernando, M. Pi, M. Barranco, W. J. Buma, and M. Drabbels, Phys. Rev. Lett. 111, 153002 (2013).

[11] D. Mateo, F. Gonzalez, and J. Eloranta, J. Phys. Chem. A 119, 2262 (2015).

[12] L. F. Gomez et al., Science 345, 906 (2014).

[13] C. F. Jones et al., Phys. Rev. B 93, 180510(R) (2016).

[14] C. Bernando, R. M. P. Tanyag, C. Jones, C. Bacellar, M. Bucher, K. R. Ferguson, D. Rupp, M. P. Ziemkiewicz, L. F. Gomez,
A. S. Chatterley, T. Gorkhover, M. Muller, J. Bozek, S. Carron, J. Kwok, S. L. Butler, T. Moller, C. Bostedt, O. Gessner, and A. F. Vilesov, Phys. Rev. B 95, 064510 (2017).

[15] D. Rupp, N. Monserud, B. Langbehn, M. Sauppe, J. Zimmermann, Y. Ovcharenko, T. Möller, F. Frassetto, L. Poletto, A. Trabattoni, F. Calegari, M. Nisoli, K. Sander, C. Peltz, M. J. J. Vrakking, T. Fennel, and A. Rouzée, Nat. Commun. 8, 493 (2017).

[16] B. Langbehn et al., Phys. Rev. Lett. 121, 255301 (2018).

[17] O. Gessner and A. F. Vilesov, Annu. Rev. Phys. Chem. 70, 173 (2019).

[18] S. M. O. O’Connell, R. M. P. Tanyag, D. Verma, C. Bernando, W. Pang, C. Bacellar, C. A. Saladrigas, J. Mahl, B. W. Toulson, Y. Kumagai, P. Walter, F. Ancilotto, M. Barranco, M. Pi, C. Bostedt, O. Gessner, and A. F. Vilesov, Phys. Rev. Lett. 124, 215301 (2020).

[19] F. Ancilotto, M. Pi, and M. Barranco, Phys. Rev. B 91, 100503(R) (2015).

[20] F. Ancilotto, M. Barranco, and M. Pi, Phys. Rev. B 97, 184515 (2018).

[21] L. F. Gomez, E. Loginov, and A. F. Vilesov, Phys. Rev. Lett. 108, 155302 (2012).

[22] E. Latimer, D. Spence, C. Feng, A. Boatwright, A. M. Ellis, and S. Yang, Nano Lett. 14, 2902 (2014).

[23] A. Volk, D. Knez, P. Thaler, A. W. Hauser, W. Grogger, F. Hofer, and W. E. Ernst, Phys. Chem. Chem. Phys. 17, 24570 (2015).

[24] F. Coppens, F. Ancilotto, M. Barranco, N. Halberstadt, and M. Pi, Phys. Chem. Chem. Phys. 19, 24805 (2017). 
[25] M. Blancafort-Jorquera, A. Vilà, and M. González, Phys. Chem. Chem. Phys. 20, 29737 (2018).

[26] F. Coppens, F. Ancilotto, M. Barranco, N. Halberstadt, and M. Pi, Phys. Chem. Chem. Phys. 21, 17423 (2019).

[27] W. E. Ernst and A. W. Hauser, Phys. Chem. Chem. Phys. 23, 7553 (2021).

[28] We remark that the term "oblate" will be used here to indicate droplets whose axial symmetry is not appreciably distorted by the vortex array they host, such as those appearing in the experiments. It is obvious that the presence of any vortex array breaks axial symmetry, and stricto sensu these droplets are not oblate.

[29] R. A. Brown and L. E. Scriven, Proc. R. Soc. London, Ser. A 371, 331 (1980).

[30] C.-J. Heine, IMA J. Numer. Anal. 26, 723 (2006).

[31] S. L. Butler, M. R. Stauffer, G. Sinha, A. Lilly, and R. J. Spiteri, J. Fluid Mech. 667, 358 (2011).

[32] K. A. Baldwin, S. L. Butler, and R. J. A. Hill, Sci. Rep. 5, 7660 (2015).

[33] H. Lamb, Hydrodynamics, 6th ed. (Dover, New York, 1945).

[34] E. Guyon, J.-P. Hulin, L. Petit, and C. D. Mitescu, Physical Hydrodynamics, 2nd ed. (Oxford University Press, Oxford, 2015).

[35] A. Bohr and B. R. Mottelson, Nuclear Structure (Benjamin, Reading, MA, 1975), Vol. 2.

[36] L. Pitaevskii and S. Stringari, Bose-Einstein Condensation and Superfluidity, International Series of Monographs on Physics Vol. 164 (Oxford University Press, Oxford, 2016).

[37] R. J. Donnelly, Quantized Vortices in Helium II (Cambridge University Press, Cambridge, 1991).

[38] G. M. Seidel and H. J. Maris, Phys. B (Amsterdam, Neth.) 194196, 577 (1994).

[39] A. L. Fetter, J. Low Temp. Phys. 16, 533 (1974).

[40] See Supplemental Material at http://link.aps.org/supplemental/ 10.1103/PhysRevB.104.094509 for videos showing the TDDFT evolution in the laboratory frame of the ${ }^{4} \mathrm{He}_{1500}$ droplet at $\Lambda=$ 0.8 for the (a) $n_{v}=0$ configuration and (b) $n_{v}=3$ configuration.

[41] M. Barranco, R. Guardiola, S. Hernández, R. Mayol, J. Navarro, and M. Pi, J. Low Temp. Phys. 142, 1 (2006).
[42] F. Ancilotto, M. Barranco, F. Coppens, J. Eloranta, N. Halberstadt, A. Hernando, D. Mateo, and M. Pi, Int. Rev. Phys. Chem. 36, 621 (2017).

[43] F. Ancilotto, M. Barranco, F. Caupin, R. Mayol, and M. Pi, Phys. Rev. B 72, 214522 (2005).

[44] M. Pi, F. Ancilotto, F. Coppens, N. Halberstadt, A. Hernando, A. Leal, D. Mateo, R. Mayol, and M. Barranco, 4HE-DFT BCNTLS, a computer package for simulating structural properties and dynamics of doped liquid helium-4 systems, https://github. com/bcntls2016/.

[45] M. Barranco, F. Coppens, N. Halberstadt, A. Hernando, A. Leal, D. Mateo, R. Mayol, and M. Pi, https://github.com/bcntls2016/ DFT-Guide/blob/master/dft-guide.pdf.

[46] J. Garaud, J. Dai, and A. J. Niemi, J. High Energy Phys. 7 (2021)157.

[47] M. Pi, F. Ancilotto, and M. Barranco, J. Chem. Phys. 152, 184111 (2020).

[48] D. V. Osborne, Proc. Phys. Soc. A 63, 909 (1950).

[49] F. Dalfovo, R. Mayol, M. Pi, and M. Barranco, Phys. Rev. Lett 85, 1028 (2000).

[50] L. J. Campbell and R. M. Ziff, Phys. Rev. B 20, 1886 (1979).

[51] F. Ancilotto, M. Pi, and M. Barranco, Phys. Rev. B 90, 174512 (2014).

[52] D. J. Tritton, Am. J. Phys. 50, 421 (1982).

[53] V. B. Eltsov, R. de Graaf, R. Hänninen, M. Krusius, R. E. Solntsev, V. S. L'vov, A. I. Golov, and P. M. Walmsley, Prog. Low Temp. Phys. 16, 45 (2009).

[54] S. K. Nemirovskii, Phys. Rep. 524, 85 (2013).

[55] J. M. Escartín, F. Ancilotto, M. Barranco, and M. Pi, Phys. Rev. B 99, 140505(R) (2019).

[56] R. P. Feynman, Prog. Low Temp. Phys. 1, 1 (1955).

[57] D. Verma, S. M. O. O'Connell, A. J. Feinberg, S. Erukala, R. M. P. Tanyag, C. Bernando, W. Pang, C. A. Saladrigas, B. W. Toulson, M. Borgwardt, N. Shivaram, M.-F. Lin, A. Al Haddad, W. Jäger, C. Bostedt, P. Walter, O. Gessner, and A. F. Vilesov, Phys. Rev. B 102, 014504 (2020).

[58] S. L. Butler, Phys. Fluids 32, 012115 (2020).

[59] M. Pi, F. Ancilotto, J. M. Escartín, R. Mayol, and M. Barranco, Phys. Rev. B 102, 060502(R) (2020). 